\title{
The Effects of a Progressive Running Program on Girls' Running Economy
}

\author{
Ryan M. Green, PhD, LAT, ATC \\ Assistant Professor \\ Southeastern Louisiana University \\ Department of Kinesiology and Health Studies \\ Hammond, LA 70402, USA \\ Holly S. Kihm, PhD, CCLS* \\ Associate Professor \\ Southeastern Louisiana University \\ Hammond, LA 70402, USA \\ Heidi Kulkin, PhD, LCSW-BACS \\ Associate Professor \\ Southeastern Louisiana University \\ Hammond, LA 70402, USA \\ Myia Graves, PhD \\ Assistant Professor \\ Department of Kinesiology \& Health Studies \\ Southeastern Louisiana University \\ USA
}

\begin{abstract}
Participation in physical activity across the lifespan is imperative for improving and maintaining good health, however, most children and youth do not participate in the recommended amount of physical activity per day. The purpose of this pilot study was to test the efficacy of a progressive running program on girls' running economy over a 14 week time period. Participants completed weekly structured running activities and completed running and strength building activities. Results showed a strong relationship between age and number of days compliant during the program and a significant relationship between the number of days participants attended lab and distance they were able to cover during midpoint and final assessments. A progressive program that is tailored specifically to youth may be a useful tool in encouraging a higher level of physical activity participation.
\end{abstract}

Keywords: Youth, Girls, Running, Physical Activity, Strategies

\section{Introduction}

Participation in physical activity across the lifespan is imperative for improving and maintaining good health. During childhood, the benefits of physical activity are many. According to the Department of Health and Human Services (DHHS), children who participate in moderate to vigorous physical activity (MVPA) tend to have higher levels of aerobic fitness, stronger muscles and bones, and a lower percentage of body fat than children who do not engage in physical activity. Other benefits of physical activity participation include improved cognition, reduced symptoms of depression, increased memory, and higher academic performance (Physical Activity Guidelines for Americans, 2019). Guidelines published by DHHS, state that children (ages 6 through 17 years) should engage in moderate and vigorous intensity physical activity for a minimum of 60 minutes each day. Activities may be broken down into small time increments and should include muscle and bone strengthening exercises in addition to aerobic activities (Physical Activity Guidelines for Americans, 2019).

One popular physical activity among children and youth is running. Many children run each year for various reasons: organized sport training, playing games with their friends, or simply to burn off some extra energy. According to Statistica, a consumer research and data company, approximately 11.04 million youth (ages 6-17 yrs) participated in running-based activities between 2006 and 2017 (Statistica, 2019). To foster enjoyment of running in youth, it is 
important to understand the components necessary to enable a child to run at a desired speed or achieve a distance goal. One overarching component that has been identified as a major determinant of distance running success is "Running Economy." (Daniels, 1985; Krahenbuhl and Williams, 1992). Running economy is the steady state oxygen required at a given speed (Krahenbuhl and Petry, 1985). For example, think of a car and gas mileage. The more economical a car, the more ground it can cover at a given speed while using the least amount of gas (energy). Different components can affect the car's economy: type of fuel for the engine, car speed, terrain, chassis maintenance, cleanliness of the engine, tire alignment, etc. With a human, movement economy can be affected by injury, running speed, running surface, strength, footwear, food/nutrition, and regular training/tissue maintenance. During childhood, running economy may be affected by growth changes. These include: stride frequency, surface area, body mass ratio, musculotendinous elastic energy storage, body composition changes, thermal responses to exercise, respiratory exchange ratio changes, anaerobic capacity, gait mechanics, and ventilatory efficiency (Rowland, 1989).

An important concept that is critical to running economy during childhood is based on Kleiber's law, or "Surface Law Theory." Briefly, the law states that an animal's metabolic rate scales to the $3 / 4$ power of the animal's mass (Kleiber, 1947). For example, a mouse has a higher metabolic rate than does a cow (rate is 10x higher in a mouse than in a cow). This higher metabolic rate helps the mouse produce more heat to stay warm. For heat production, having a higher metabolic rate is good as it will help keep the body warm.

In relation to children, the smaller the body (i.e. less mass), the higher the metabolic rate (i.e. higher respirations and higher heart rates). While this is essential for keeping the body warm and sustaining life, it is not helpful with regards to running economy. Higher heart rates and higher respirations requires the body to use more energy to sustain itself. Lower heart rates and lower respiration rates requires less energy expenditure. Rowland (1989) surmised that "smaller individuals have some built-in impairment in efficiency of muscle contraction such that for a given work output a greater amount of energy is required and more heat is produced compared to an older [bigger] individual. Muscular effort to move one kilogram of body mass at a given speed will require more [energy] in the child because metabolism is geared to surface area instead of weight" (Rowland, 1989: 320). When a child is born, their metabolic rate is very high in relation to an adult's metabolic rate. As a child develops, their metabolic rate decreases while their surface area increases. The increase in surface area allows for more heat dissipation resulting in a lower heart rate and lower respiratory rate therefore becoming more economical.

Theoretically, economy should increase with age until the end of puberty when the body's growth is complete. To further increase economy, children should participate in additional activities that support aerobic fitness and muscle and bone strength. Some research points to the benefits of repeated bouts of high intensity plyometric exercises to enhance aerobic and anaerobic fitness in children as that is the way they naturally play (Faigenbaum et al., 2009). However, other studies recommend that regular and specialized distance running not begin until the early stages of puberty (ages 11-13) because children lack many of the hormones that allow for training adaptations in the musculoskeletal system and they are poorly suited for longer bouts of intense exercise (Krahenbuhl and Petry, 1985; Krahenbuhl and Williams, 1992). Until puberty, however, it has been suggested that children may benefit from a comprehensive fitness that included technique instruction on sprinting and stretching to enhance their overall fitness (Faigenbaum et al., 2009).

Every aspect of sports or athletics involves some level of training. In golf it is skill training in swinging the club through the same movement pattern, in football it is power training to move large, heavy objects, and in running the training involves moving over distance or at speed, or sometimes both. This training can be haphazard in its construction in hopes of achieving a desired outcome or it can be calculated, well-thought out progression where the end result has a better chance of achievement. In order for the body to continue to positively adapt to the strain put upon it and reduce the chance of injury, a logical progression of ever-increasing loads may yield the greatest change without injury, burnout, or the negative effects of overtraining (Benjamin et al., 2014; Kemper, 2000; Davis et al., 2019).

Though there is literature supporting the idea of integration of neuromuscular and strength training in youth (Faigenbaum and McFarland, 2006; Faigenbaum et al., 2011), there is limited research on the effects of progressive running programs in children, especially in preadolescent girls (Krahenbuhl and Petry, 1985; Krahenbuhl and Williams, 1992). The purpose of this pilot study was to test the efficacy of a progressive running program on girls' running economy over a 14 week time period. Participants completed weekly structured running activities and completed running and strength building activities (i.e. squats, push-ups, planks, planks with a twist, lunges) throughout the week while at home. The results from the study may serve as guidance for physical education teachers and public health professionals working with children to increase their physical activity participation through running and across the lifespan. 


\section{Methods}

\subsection{Participants}

Seven girls between the ages of 8 and 12 years attending an elementary school in southeastern Louisiana were recruited and enrolled in an after-school exercise program located at a local university laboratory (Kihm, Sandoval, and Staiano, 2017). This elementary school was chosen because of the relationship between the college faculty and the physical educators and parents of the participants. Prior to engaging in study-related activities, staff members obtained written consent from parents and verbal informed assent from participants who were under the age of 10 years. Participants 10 years and over provided written informed assent. The study protocol and all other study-related materials and handouts were approved by the institutional review board.

\subsection{Instruments}

Quantitative data collected for the running activities were recorded on data sheets by the undergraduate students and then handed in to the lab graduate assistant at the end of each session. Distance, measured in steps, completed during sessions was recorded using a fitness tracker (Fit Bit Charge). Time completed was measured with a stopwatch.

\subsection{Procedure}

Program sessions were held either outside on the University's track or inside in the gymnasium depending on weather. Undergraduate students collected data while supervised by the Principal Investigators. The undergraduate students enrolled in a research methods course and received 2 weeks of training on the protocol, and were also trained and certified in research methodology by the Collaborative Institutional Training Initiative. Participants attended the program once per week after school for approximately two hours, and were asked to complete one five-minute strength building session and one running session each day for the remainder of the week while at home. Participants indicated completion of the at-home sessions in personal journals that were turned in each week to the Principal Investigators.

During each session, participants completed a 10 minute warm-up activity, 20 minute running activity, and a 10 minute cool down activity (Table 1). Remaining meeting time was spent discussing various topics related to health and wellness including proper nutrition, flexibility, and strength training. During the 20 minute running activity, the undergraduate students monitored and recorded each participant's distance completed.

\section{Results}

\subsection{Data Analysis}

Data were analyzed using the Statistical Package for Social Sciences (SPSS). Four hypotheses were tested using a paired samples t-test analysis and 10 hypotheses were tested using Pearson's bivariate correlations.

T-test

The following hypotheses were tested using a paired samples t-test analysis:

- There will be a significant mean difference between pretest and midpoint scores in terms of number of steps.

- There will be a significant mean difference between pretest and posttest scores in terms of number of steps.

- There will be a significant mean difference between midpoint and posttest scores in terms of number of steps.

- There will be a significant mean difference between pretest BMI score and posttest BMI score.

\section{Results}

Of the 7 participants in this study, 29\% ( $\mathrm{n}=2)$ of participants were African American and $71 \%(\mathrm{n}=5)$ were Caucasian. Participant ages ranged from 8 to12 years with a mean age of 10 years $(\mathrm{SD}=1.15)$. The number of days compliant reported by participants ranged from 9 days to 74 days during the 14 week program. Of the participants in this study, 43\% ( $\mathrm{n}=3)$ reported attending 11 labs, 14\% $(\mathrm{n}=1)$ reported attending 10 labs, 29\% (n=2) reported attending 7 labs, while $14 \%(n=1)$ reported attending 6 labs. Seventy-one percent $(n=5)$ of participants reported their weights in the healthy weight range, while $29 \%(\mathrm{n}=2)$ reported their weights in the overweight range. Pretest BMI scores ranged from 17.33 to 31.40 with a mean of $24.21(\mathrm{SD}=5.16)$ and posttest BMI scores ranged from 17.60 to 32.30 with a mean of 24.19 (SD = 5.01). Pretest, midpoint, and posttest scores were based on the actual number of steps taken by each participant. Pretest scores ranged from 2248.00 to 2656.00 with a mean of 2414.57 (SD $=155.90)$. Midpoint scores ranged from 2376.00 to 2792 with a mean of 2624.86 ( $\mathrm{SD}=159.90)$. Posttest scores ranged from 2852.00 to 3706.00 with a mean of $3446.00(\mathrm{SD}=285.08)$.

Data were analyzed using the Statistical Package for Social Sciences (SPSS). Four hypotheses were tested using a paired samples t-test analysis and 10 hypotheses were tested using Pearson's bivariate correlations. 
The following hypotheses were tested using a paired samples t-test analysis:

- There will be a significant mean difference between pretest and midpoint scores in terms of number of steps.

- There will be a significant mean difference between pretest and posttest scores in terms of number of steps.

- There will be a significant mean difference between midpoint and posttest scores in terms of number of steps.

- There will be a significant mean difference between pretest BMI score and posttest BMI score.

As depicted in Table 2, of the 4 sets of pairs analyzed in SPSS using a paired samples t-test analysis, 3 pairs boasted significant results.

\section{Bivariate Correlations}

As aforementioned, in addition to the paired samples t-test analysis, the following hypotheses were tested using bivariate Pearson's correlations:

- There will be a significant relationship between age and pretest scores.

- There will be a significant relationship between age and midpoint scores.

- There will be a significant relationship between age and posttest scores.

- There will be a significant relationship between age and number of days participants attended lab.

- There will be a significant relationship between age and number of days compliant during the program.

- There will be a significant relationship between midpoint scores and number of days compliant during the program.

- There will be a significant relationship between posttest scores and number of days compliant during the program.

- There will be a significant relationship between midpoint scores and number of days participants attended lab.

- There will be a significant relationship between posttest scores and number of days participants attended lab.

- There will be a significant relationship between number of labs attended and number of days compliant during the program.

Of the 10 hypotheses analyzed in SPSS using bivariate correlations, 2 hypotheses were significant. The study indicated that there was a strong positive significant relationship between age and number of days compliant during the program $(\mathrm{r}=.75, p=.05)$. That is, as a participant's age increased, so did the number of days compliant in the program. Additionally, the researchers found that the relationship between the number of days participants attended lab and midpoint scores was significant and demonstrated a strong positive association $(\mathrm{r}=.87, p=.01)$. This indicated that as the number of days participants attended lab increased, so did midpoint step scores.

\section{Discussion}

In this observation of children's running economy, these findings have important practical relevance for designing children's running programs. Since cardiovascular endurance is an important component of health-related fitness assessments in elementary school students, promoting health and physical wellness through running may be an integral aspect of achieving optimal well-being for future generations (Marttinen et al., 2017). While there were no significant changes in health characteristics (BMI) over the length of the program, there were a significant number of steps taken (pre- vs. post-test scores) that may indicate a trend towards better economy of movement and thus better health markers over the long-term. Over 1,000 more steps were taken in the post-test assessment than in the pre-test assessment.

As is suggested here, running economy in children may relate to the Surface Law Theory proposed by Kleiber (1947). A younger student with more mass is at a double disadvantage. The first disadvantage is that it takes more effort to move more mass and the second disadvantage is that children are less economical when they are younger because they have not figured out the most effective pathways of movement. When a participant has less body mass (less muscular effort to move), that is an advantage in running. However, when individuals are younger, they tend to be less economical because they have not had the time to fully develop movement pathways. Older participants should be more economical with age, but since they may be carrying more body mass, it takes more muscular effort to move them. Finally, a leaner, older student has become the most economical due to natural movement progression and she is leaner and therefore requires less muscular effort to move her mass.

The purpose of this pilot study was to test the efficacy of a progressive running program on girls' running economy over a 14 week time period. While 14 weeks may not seem like a long time for a child to produce significant economic changes in their running patterns (Krahenbuhl and Petray, 1985), studies indicate that children become more economical simply by remaining active through their development and not undergoing a specialized running technique training program regardless of how active they are (Krahenbuhl and Williams, 1992). They stated, "Long-term participation in running training may augment improvements in running economy that come naturally with age" (Krahenbuhl and Williams, 1992: 462). Older children completed more at-home activities so they may have had more opportunities to develop neural pathways and become more economical. 
When one has more running economy, there is fluidity of movement, less injury, more running since there is less injury to hamper the running, more endurance, and more steps.

The current program did not focus on teaching running technique as a means to becoming more economical, but simply encouraged the participants to move most days of the week. The running progression program guided participants and their guardians with simple, logical "steps" to get movement in their life even outside of the scheduled lab sessions. When the number of days participants attended lab increased, their midpoint scores for number of steps increased as well. As participants continued to move over the course of the program, the children may have figured out their most economical, "preferred movement pattern" and were able to obtain more steps during a test session (Baltich et al., 2015; Anderssen et al., 2009; Faigenbaum et al., 2011).

Another subtle finding noted was that the younger participants had a slightly lower average of overall steps compared to older participants ( 2700 vs 2731 ). This finding supports the idea proposed by (Rowland, 1989: 465) who stated that "metabolic cost of respiration is greater in children and suggested that this may contribute to poorer economy in younger subjects". In a future, larger study, it is anticipated that younger children would complete significantly fewer steps per exercise session than older children.

\subsection{Limitations}

Because this was a small, pilot study several limitations have been identified. First, the study was comprised of only elementary age girls. The sample was one of convenience and future studies should include more participants of varied ages. Second, the study focused only on female students. Results cannot be confidently applied to other populations outside of the test population without some interpretation. Furthermore, participants self-reported their at-home activity levels. Though participants shared they understood the importance of reporting accurate information, regardless how compliant they were to the program, some participants may have inaccurately recorded their activity. Future studies should address these limitations by studying youth boys as well as older age brackets of both genders, employ more concise measures of at-home activity via activity trackers or the like, and give subjects activity options for physical engagement to study their physical literacy and well-being.

\subsection{Conclusion}

Regular participation in a progressive running program does improve running economy in 8-12 year old girls. These findings can be leading steps in assisting health and physical educators to determining the most effective means to get youth girls moving in an economical manner now and for years to come. An important aspect of any structured or unstructured physical activity program is to ensure participants understand two key concepts: physical activity can be enjoyed by all participants, regardless of their current fitness level, and physical activity is not exclusively competitionbased. Many times youth who have very little fitness do not feel motivated to participate in programs due to a fear of "finishing last" or not being able to keep up with the group. This is where a progressive running program, tailored to individual participants may be extremely beneficial. All participants essentially "start where they are at" and then work toward a mutual goal. Helping participants understand they are capable of increasing their physical fitness and giving them the tools they need to do so, will provide a life-long path to wellness.

\section{References}

Anderssen N, Kjonniksen L, and Wold B (2009) Organized youth sport as a predictor of physical activity in adulthood. Scandiavian Journal of Medicine and Science in Sports 19: 646-654.

Baltich J, Hoerzer S, Nigg BM, et al. (2015) Running shoes and running injuries: mythbusting and a proposal for two new paradigms: 'preferred movement path' andcomfort filter'. British Journal of Sports Medicine 49(20): $1290-1294$.

Benjamin HJ, Brenner JS, DiFiori JP, et al. (2014) Overuse injuries and burnout in youth sports: a position statement from the American Medical Society for Sports Medicine. British Journal of Sports Medicine 48: 287-288.

Daniels JT (1985) A physiologist's view of running economy. Medicine and Science in Sports and Exercise 17(3): 332-338.

Davis IS, Krabek BJ, Tenforde AS, et al. (2019) Youth distance running: strategies for training and injury reduction. Current Sports Medicine Reports 18: 53-59.

Faigenbaum AD, Farrell AC, Radler T, et al. (2009) Plyo Play: A novel program of short bouts of moderate and high intensity exercise improves physical fitness in elementary children. The Physical Educator 66(1): 37-44.

Faigenbaum AD, Ford KR, Myer GD, et al. (2011) When to initiate integrative neuromuscular training to reduce sports-related injuries in youth? Current Sports Medicine Reports 10: 155-166. 
Faigenbaum AD and McFarland J (2006) Make time for less intense training. Strength and Conditioning Journal 28: $77-79$.

Kemper HC (2000). Skeletal development during childhood and adolescence and the effectsof physical activity. Pediatric Exercise Science 12: 198-216.

Kihm H, Sandoval P, and Staiano AE (2017) Project IPAL: To enhance the well-being of elementary school children. Journal of Family and Consumer Sciences 109(1): 54-56.

Kleiber M (1947) Body size and metabolic rate. Physiological Reviews 27(4): 511-41.

Krahenbuhl GS and Petry CK (1985) Running training, instruction on running technique, and running economy in 10year old males. Research Quarterly for Exercise and Sport 56(3): 251-255.

Krahenbuhl GS and Williams TJ (1992) Running economy: changes with age during childhood and adolescence. Medicine and Science in Sports and Exercise 24: 462-466.

Marttinen R, Mercier K, and Phillips SR (2017) Fitness Assessment: Recommendations foran Enjoyable Student Experience. Strategies 30(5): 19-24. DOI: 10.1080/08924562.2017.1344168.

Physical Activity Guidelines for Americans. (2019). 2nd ed. [ebook] Washingtion, DC: U.S. Department of Health and Human Services, pp.105-106. Available at: https://health.gov/paguidelines/second-edition/pdf/Physical_Activity_Guidelines_2nd_edition.pdf [Accessed 5 Aug. 2019].

Rowland TW (1989) Oxygen uptake and endurance fitness in children: a developmental Perspective. Pediatric Exercise Science 1: 313-328.

Statista (2019). Number of youth participants in running in the United States from 2006-2017 (imillions). Available at: www.statista.com/statistics/190738/youth-participants-in-running-in-the-us-since-2006/ (accessed 5 August 2019). 